\title{
MESO-MACRO MODELLING OF CRACK-INDUCED DIFFUSIVITY IN HETEROGENEOUS MATERIALS
}

\author{
N. BENKEMOUN, O. AMIRI \\ Université Nantes Angers Le Mans (L'UNAM), GeM, Research Institute of Civil Engineering and Mechanics, \\ CNRS UMR 6183, Nantes University, IUT Saint-Nazaire 58 rue Michel Ange, 44600 Saint-Nazaire, \\ FRANCE \\ e-mail: nathan.benkemoun@univ-nantes.fr
}

Key words: Durability, Meso-macro modelling, Chloride ions transport, Strong discontinuity approach

\begin{abstract}
This paper presents a meso-macro numerical approach for the determination of macroscopic diffusivity tensors in heterogeneous materials such as concrete. These macroscopic tensors account for important features of heterogeneous materials: (1) cracking process (evolution from diffuse cracks in the bulk to localized macro-crack), (2) tortuosity and connectivity of the crack, (3) induced-anisotropy and (4) presence of aggregates. We present numerical examples in the context of concrete-like materials. We show how the crack pattern and the presence of aggregates induce the anisotropy of the macroscopic diffusivity tensor.
\end{abstract}




\section{INTRODUCTION}

Regarding reinforced concrete civil engineering facilities, some corrosive external agents, sea water and spray, - are susceptible to penetrate cement materials and deteriorate its mechanical performances. Consequently, because of deteriorated mechanical performances, the structure durability and service life are affected. The most corrosive penetrating agents with regards to concrete are chloride ions. A considerable amount of effort has been devoted to the modelling of chloride ions transport mechanisms (diffusion, migration, convection) and behaviour within cement materials. However, chloride transport modelling is reliable only if the main degradation factor of concrete structures into service, i.e., cracking, is considered (see ([6]) and ([7])).

A survey of the literature reveals that during the last couple of years, some research work has been devoted to the numerical simulation of the coupling ${ }^{\dagger}$ between chloride ion transport and concrete crack formation mechanisms. Concerning the coupling, researchers link an equivalent diffusion coefficient $D_{e}$ to a crack width parameter. They propose models depending on this coefficient fitted in experimental data (see ([11]), ([6]) and ([8])). ([11]) and ([6]) suggest a linear relationship between the crack width and the equivalent diffusion coefficient $D_{e}$. They show that only over a threshold crack width value of $80 \mu \mathrm{m}$, the diffusion coefficient starts to increase. In addition, in ([6]), a important step forward is made: the authors propose a relationship between the crack width and the diffusion coefficient through the crack $D_{c r}$. This information is of great interest for the mesoscale coupled approach detailed in this paper. In ([5]), the author proposes an equivalent diffusion coefficient of a power function of the crack width. The author finds that for a crack width higher than $135 \mu \mathrm{m}$ the equivalent diffusion coefficient increases rapidly.

Regarding the numerical simulation of this coupling, some studies are particularly relevant. In ([12]), the authors integrate the diffusion coefficient through the crack obtained in ([6]) in their numerical model of diffusion based on the
FE method. Then they compare their numerical results with the experimental ones of ([10]). Note that in ([12]), only artificial cracks are considered (no mechanical simulations are performed). ([29]) consider a lattice-type model (see ([9]) for details) as numerical model of diffusion. They fit in the experimental results of ([10]) to find an equivalent diffusion coefficient. Then ([29]) consider a mesoscale lattice-type model with three phases (ITZ, aggregates, mortar) in order to gauge the influence of the ITZ and the aggregates on the depth of chloride penetration. Here again only artificials crack are taken into account. In ([4]) and ([3]), the authors propose a numerical coupling between two models: for mechanical and diffusion simulations respectively, the Delft lattice model (see ([23]) for details) and the transport lattice model (see ([4]) for details). Contrary to the prior numerical works, mechanical simulations are performed leading to real cracks. In ([3]), according to findings of ([34]), the authors impose a chloride concentration only on cracks wider than $12 \mu \mathrm{m}$ obtained after the numerical simulation of a splitting test. In ([4]), the authors assess their numerical model with the experimental results of ([5]) and ([10]) for cracked mortar samples by considering $D_{c r}$ of ([6]).

However, regarding this literature, none of the models are capable of giving macroscopic quantities depending on the cracked state of concrete and more globally on its heterogeneous aspect. This limitation may be due to: either because they do not consider real cracks and thus avoid connectivity, tortuosity aspects of the crack pattern and crack width heterogeneities in the computation and/or either because they consider an equivalent diffusion coefficient $D_{e}$ that can be seen as a phenomenological variable such as damage or plasticty in mechanics ([22]). In this case, $D_{e}$ does not account explicitely of the crack pattern failure and the heterogeneous aspect of the material.

From a civil engineering point of view, this information is crucial regarding durability and service-life problems of civil engineering facilities. We propose in this paper a method based 
on an upscaling process in order to provide a macroscale diffusivity (mean diffusivity tensor) accounting for the heterogeneous mesoscale structure of concrete, and correlate to the crack pattern and to the crack width values of numericallyinduced cracks. In this context, important features of the cracking process are taken into account in the macroscopic diffusivity: (1) the evolution from diffuse cracks in the bulk to localized macro-crack(s), (2) the tortuosity of the crack and (3) the induced-anisotropy. To the author's knowledge, only the work of ([17]) provides such macroscopic information.

In this paper, we model concrete at the mesoscale as an heterogeneous quasi-brittle two-phase material based upon the work of ([1]). This model is presented in Section 2. In Section 3, we introduce a simple chlorides ions transport model relying on Fick's law. The mesoscale coupling is then examined. In Section 4, the upscaling method for the determination of the mean diffusivity tensor is presented, based upon the work of ([20]). Finally, in Section 5, we show the capability of the approach to determine mean diffusivity tensors in the context of concrete-like materials.

\section{MECHANICAL MODEL: TWO-PHASE QUASI-BRITLLE MATERIAL}

In this section, we give a brief description of the mechanical model. For the readers interested in more details, a complete description of the model, its numerical implementation and a number of illustrative examples of the model predictive capabilities can be found in [1].

\subsection{Meso-model features}

The numerical model for the mechanical simulations is based upon a two-phase (stiff aggregates embedded into a mortar matrix) quasi-brittle model capable of representing the behavior of concrete-like materials under complex loading paths. In order to take into account the influence of the shape, the size, the distribution and the mechanical properties of aggregates on the mechanical behavior of concrete, the mesoscale ([32], [2]) is chosen to be the scale of computa- tion. The numerical approach we work with, at the mesoscale, is based upon a 3D lattice finite element model ([25], [24], [33] and [14]) whose truss elements kinematics is enhanced by two discontinuities.

The first discontinuity is a weak discontinuity - continuous displacement field and discontinuous strain field, ([18]) - introduced because of the non-adaptated meshing process ([16]). This process consists in a unique homogeneous mesh whose nodes are placed independently from the morphology of the aggregates. A significant amount of computation time is saved at this stage. However, some truss elements are cut into two parts, each having different elastic properties. That's why in order to take into account this special kinematics in the truss elements, this weak discontinuity is introduced.

The second discontinuity is a strong discontinuity - discontinuous displacement field and unbounded strain field, ([27]) - introduced in order to represent micro-cracks that may occur in any of different phases (aggregates or mortar matrix for two-phase materials) and to capture the interface failure (debonding). Moreover, the key point pertains to strong discontinuities capability to model softening behavior without any mesh dependency which is the major issue dealing with failure of quasi-brittle materials.

The weak discontinuity is present only for the truss elements split into two parts, each having a different Young modulus. The strong discontinuity is introduced by means of a yield function $\Phi$ which is triggered only in traction. Thus two constitutive models appear for a truss element: a continuum one (outside the discontinuity) which is elastic (see Figure 1(a)), and a discrete one (over the discontinuity) which is quasi-brittle (see Figure 1(b)). We denote by $t_{\Gamma}$ the traction vector over the discontinuity and $[|u|]$ the crack width which belongs to the set of unknowns.

The yield function is such as:

$$
\Phi=t_{\Gamma}-\left(\sigma_{u}-q\right)
$$




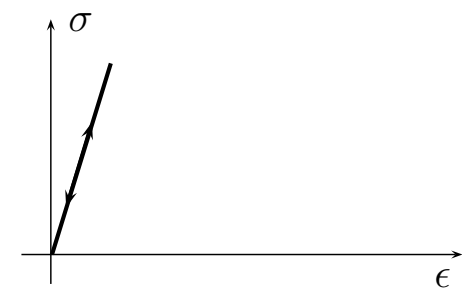

(a) behavior outside the discontinuity

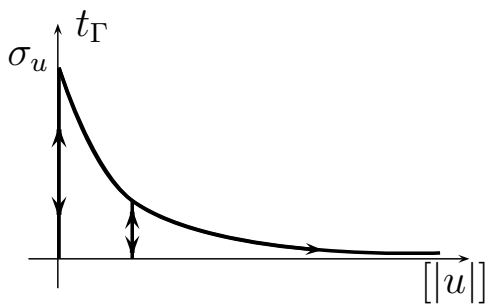

(b) behavior at the discontinuity

Figure 1: Elastic-quasi-brittle behavior

where $q$ is the stress-like variable

$$
\begin{aligned}
q & =\mathrm{k}([|u|]) \\
\text { with } \mathrm{k}([|u|]) & =\sigma_{u}\left(1-\exp \left(-[|u|] \frac{\sigma_{u}}{G_{f}}\right)\right) .
\end{aligned}
$$

In summary, there are altogether eight model parameters: the Young modulus $E^{\oplus}$ for the mortar matrix and $E^{\ominus}$ for aggregates, for the continuum model and the ultimate tensile strength before softening, $\sigma_{u_{i}}$ and the fracture energy, $G_{f_{i}}(i=1,2,3$ for respectively the mortar matrix, aggregates and interfaces) for the discrete model. We note $G_{f_{i}}$ the area under the curve $t_{\Gamma}-[|u|]$.

\subsection{Mathematical framework}

Having at end the weak and strong discontinuities, the total strain is written in the context of the EAS (Enhanced Assumed Strain, [28]) method such as:

$$
\varepsilon=\underbrace{\nabla^{s} \bar{u}}_{\text {regular }}+\underbrace{\tilde{\varepsilon}}_{\text {weak }}+\underbrace{\hat{\varepsilon}}_{\text {strong }}
$$

where $\nabla^{s} \overline{\boldsymbol{u}}$ is the symmetric gradient of the displacement field. As in ([28]), we refer to $\tilde{\varepsilon}$ and $\hat{\varepsilon}$ as the enhanced parts of the strain field. The notation $\bullet$ (resp. $\hat{\bullet})$ refers to weak (resp. strong) discontinuity.
In the context of a truss element, $\tilde{\varepsilon}$ and $\hat{\varepsilon}$ have the following form:

$$
\tilde{\varepsilon}=\boldsymbol{G}_{\mathrm{w}}^{\oplus / \ominus}[|\epsilon|] \quad \text { and } \quad \hat{\varepsilon}=\boldsymbol{G}_{\mathrm{s}}[|u|]
$$

where $\boldsymbol{G}_{\mathrm{w}}^{\oplus / \ominus}$ and $\boldsymbol{G}_{\mathrm{s}}$ are enhanced functions. $[|\epsilon|]$ and $[|u|]$ are the enhanced interpolation parameters and belong to the set of unknowns.

This strain field (equation (3)) is then introduced in the $\mathrm{Hu}$-Whasizu variational formulation ([30]) leading to the FE problem to be solved in terms of the displacement field $\boldsymbol{d}$ and the enhanced interpolation parameters $[|\epsilon|]$ and $[|u|]$ for the weak and strong discontinuities, respectively. The solving procedure is achieved by a local-global solving process: $[|\epsilon|]$ and $[|u|]$ are computed by means of a return mapping algorithm ([26]) and after a static condensation of $[|\epsilon|]$ and $[|u|]$ ([31]), the displacement field $\boldsymbol{d}$ is computed for each iteration $k+1$ of a typical time step $n+1$.

\subsection{Important feature for the mesoscale cou- pling procedure}

We recall that $[|u|]$ is nothing but the crack width value. It is quite clear that such an information is crucial for the computation of mass transport within mesoscale cracks and thus to achieve fine couplings at the mesoscale. In addition, the set of cracks orientations clearly gather the whole information dealing with the fracture pattern anisotropy inducing the anisotropy of the macroscopic diffusivity. These two important features (crack width values and crack orientations) are the key points for the mesoscale coupled approach between the mechanical model and the chlorides ions transport model. This coupled approach is detailed in the next section.

\section{MESOSCALE COUPLED APPROACH}

As presented in Section 2, the mechanical model is based upon the Strong Discontinuity Approach (SDA). This leads to the representation, in terms of displacement jump, of the fine scale cracks and to the computation of this jump as the crack width $[|u|]$. Thus at the end of each mechanical time step, the crack width values for 
each finite element are given by the mechanical model. These crack width values are then used as input data in the chlorides ions transport problem leading to a weak coupling at the mesoscale with the mechanical problem. In this Section, we first present the chloride ions transport model retained in this work and then show how the aforementioned weak coupling is performed.

\subsection{Chloride ions transport model}

Transport by diffusion, resulting of a difference of concentration in various zones, has been retained for the chloride ions transport model. Thus it relies on Fick's law such as:

$$
\vec{q}(\vec{x})=-D_{m} \cdot \vec{\nabla} c(\vec{x}),
$$

where $\vec{q}(\vec{x})$ is the mass flux $\left[\mathrm{kg} /\left(\mathrm{m}^{2} s\right)\right], D_{m}$ the mesoscale diffusion coefficient $\left[\mathrm{m}^{2} / \mathrm{s}\right], \vec{\nabla}$ the gradient operator $[1 / m]$ and $c(\vec{x})$ the mass concentration $\left[\mathrm{kg} / \mathrm{m}^{3}\right]$.

Remark: Chloride ions transport simulations are performed considering the same mesh as for mechanical simulations, namely a 3D lattice finite element model. Equation (5) has therefore a $1 \mathrm{D}$ form.

Injecting equation (5) in the mass balance equation ([19]) and considering the aforesaid remark, Fick's second law is obtained such as:

$$
\frac{\partial c}{\partial t}=-D_{m} \cdot \frac{\partial^{2} c}{\partial x^{2}}
$$

Equation (6) represents the problem to be solved in terms of mass concentration field. The resolution is achieved by means of the Finite Element Method ([15]) leading the following discretised form of (6):

$$
\left[K+\frac{M}{\Delta t}\right]_{n+1}^{(k)} \Delta c_{n+1}^{(k+1)}=-R_{n+1}^{(k)},
$$

where $M$ is the mass matrix, $K$ the diffusion matrix and $R$ the residual. A Euler-backward integration scheme is used for the time dependent term.

In the next section, we expose how the coupling with the mechanical problem is performed. As suggested in the introduction, it is based upon the experimental work of ([6]).

\subsection{Mesoscale coupled approach through ([6])}

In the present paper, the mesoscale coupling is addressed by considering the mesoscale diffusion coefficient $D_{m}$ as a function of the crack width $[|u|]$. This consideration relies on the experimental results of ([6]). The authors provide a relation between the diffusion coefficient through the crack $D_{c r}$ and the crack width $[|u|]$ such as:

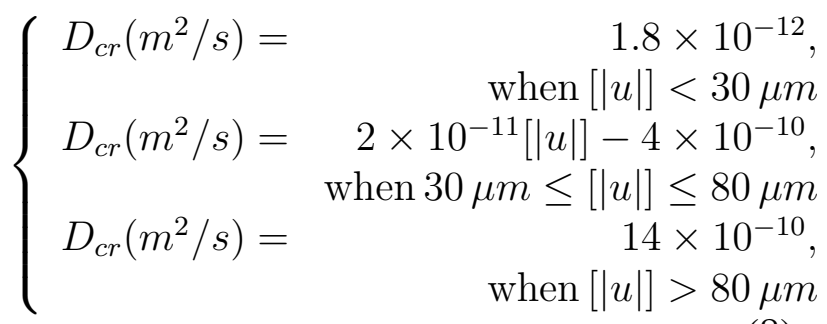

Finally, concerning the coupling, it is as follows: in a first time, mechanical simulations are performed by means of the model presented in Section 2. At the end of each mechanical time step, the crack width values for each finite element are computed. In a second time, chloride ions transport simulations are performed with these crack width values used as input data in the mesoscale diffusion coefficient $D_{m}$ with the form of (8). The models are therefore chained and not explicitly coupled.

Having at end the mesoscale coupling, we now turn to the upscaling method leading to the computation of the macroscopic diffusivity tensor (mean diffusivity tensor).

\section{UPSCALING METHOD: MEAN DIFFU- SIVITY TENSOR COMPUTATION}

In the former Section, the mesoscale coupled approach between the mechanical and chlorides ions transport models was presented. In this Section focus is made on a upscaling method leading to the computation of the macroscopic diffusivity tensor. Thus at the end of this Section, a meso-macro numerical approach accounting for crack-induced diffusivity in heterogeneous materials will be built. 


\subsection{Mean gradient of concentration and mean flux}

We define the mean concentration gradient $\vec{G}\left[\mathrm{~kg} / \mathrm{m}^{4}\right]$ and the mean flux $\vec{Q}\left[\mathrm{~kg} /\left(\mathrm{m}^{2} \mathrm{~s}\right)\right]$ within a domain $\Omega$ by the following relationship:

$$
\begin{aligned}
\vec{G} & =\frac{1}{V} \int_{\Omega} \vec{\nabla} c(\vec{x}) d \Omega, \\
\vec{Q} & =\frac{1}{V} \int_{\Omega} \vec{q}(\vec{x}) d \Omega,
\end{aligned}
$$

where $V$ is the volume of $\Omega$. We remind that $\vec{q}(\vec{x})$ is Fick's velocity at point $\vec{x}$ and $\vec{\nabla} c(\vec{x})$ is the concentration gradient at this point such as the Fick's law gives:

$$
\vec{q}(\vec{x})=-D_{m} \cdot \vec{\nabla} c(\vec{x}),
$$

where $D_{m}$ is the mesoscale diffusion coefficient with the form of equation (8).

In order to determine the mean diffusivity tensor, the authors follow the method proposed in ([20]) and ([21]) for hydraulic transport problem (incompressible flow) in heterogeneous media and developped for gas transport problem (compressible flow) in cracked media such as concrete in ([13]). Herein we extend the method to chlorides ions transport problem in heterogeneous media. Note that the term heterogeneous gathers both inclusions such as aggregates in concrete and cracks. The mean concentration gradient $\vec{G}$ and the mean flux $\vec{Q}$ are computed from the values of concentration and flux on the frontier $\partial \Omega$ of the domain $\Omega$ such as:

$$
\begin{aligned}
\vec{G} & =\frac{1}{V} \int_{\partial \Omega} c(\vec{x}) \vec{n}(\vec{x}) d S, \\
\vec{Q} & =\frac{1}{V} \int_{\partial \Omega}(\vec{q} \cdot \vec{n}) \vec{x} d S,
\end{aligned}
$$

where $\vec{n}$ is the outward unit vector from the surface $S$ and $d S$ is a surface element of $\partial \Omega$.

As we'll see hereafter, equations (12) and (13) are well adaptated for numerical simulations and their form provide an efficient way to compute the mean diffusivity tensor when boundary conditions are applied. These equations respectively represent the concentration gradient and the flux computed from the values of concentration and flux on the contour for any heterogeneous media - presence of cracks and/or inclusions -, any boundary condition - Dirichlet or Neumann-type - and, any frontier shape.

\subsection{Boundary conditions: linear concentra- tion}

In the present paper, Dirichlet-type boundary conditions are assumed. We consider condition of linear pressure at the contour such as:

$$
c(\vec{x})=\vec{A} \cdot \vec{x}+c_{0}, \quad \forall \vec{x} \in \partial \Omega,
$$

where $\vec{A}$ is a constant vector and $c_{0}$ a constant scalar.

Following the development made in ([20]) for hydraulic transport problem under the condition of linear pressure at the contour, we obtain: the equality $\vec{G}=\vec{A}$ and a direct link between the mean concentration gradient $\vec{G}$ and the mean flux $\vec{Q}$ such as:

$$
\vec{Q}=-\underline{\underline{D}} \cdot \vec{G},
$$

where $\underline{\mathrm{D}}$ represents the mean diffusivity tensor of the domain $\Omega$.

Incorporing the equality $\vec{G}=\vec{A}$ in (15) yields:

$$
\vec{Q}=-\underline{\underline{D}} \cdot \vec{A}
$$

Finally, equation (16) provides a straightforward way for the numerical computation of the mean diffusivity tensor $\underline{\underline{\mathrm{D}}}$. Indeed, the nine components of the tensor $\underline{\underline{\underline{D}}}$ are obtained by computing $\vec{Q}$ for three distincts directions of the vector $\vec{A}$ present in (14). These directions are shown in Figure 2 for a $100 \times 100 \times 100 \mathrm{~mm}$ cubic domain: Figure 2(a) corresponds to $\mathrm{X}$ direction, Figure 2(b) to the $\mathrm{Y}$ direction and Figure 2(c) to the $\mathrm{Z}$ direction of the domain.

As demonstrated in ([21]), two properties of $\underline{\mathrm{D}}$ are worth noting: it is symmetric and positivedefinite. These properties as well as the implementation of the method will be assessed in the next section by means of numerical examples. 


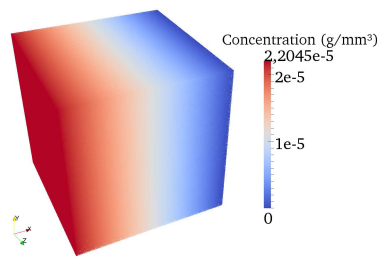

(a)

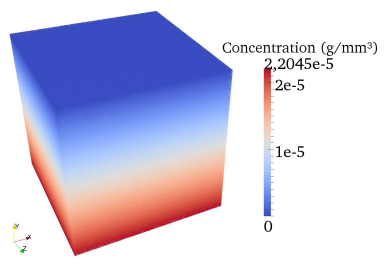

(b)

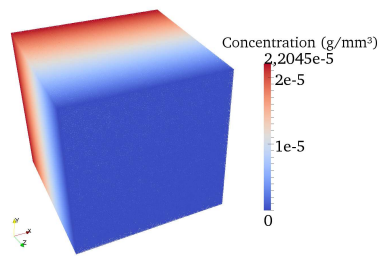

(c)

Figure 2: Condition of linear pressure on the contour in the $\mathrm{X}, \mathrm{Y}$ and $\mathrm{Z}$ directions

\section{NUMERICAL EXAMPLES}

In this Section, the upscaling method presented in the previous section is illustrated by means of numerical examples. We consider concretelike materials with two types of heterogeneities (cracks and/or aggregates). We show how the anisotropy of the mean diffusivity tensor $\underline{\underline{D}}$ is induced by the the failure pattern anisotropy.

\subsection{Artificially cracked domain}

The first example deals with a $100 \times 100 \times 100$ $\mathrm{mm}$ cubic domain cut by a perfect plane crack. The crack is located in the plane $\mathrm{Y}=50 \mathrm{~mm}$. The value of the crack width $[|u|]$ is imposed, ranging from 0 to $500 \mu \mathrm{m}$. For each value of the crack width, the method presented up above is applied ; the nine components of the mean diffusivity tensor are computed. We remind that the mesoscale coupling is performed by means of the experimental law of ([6]) presented in Section 3.

Figure 3 shows the position of the crack in the cubic domain.

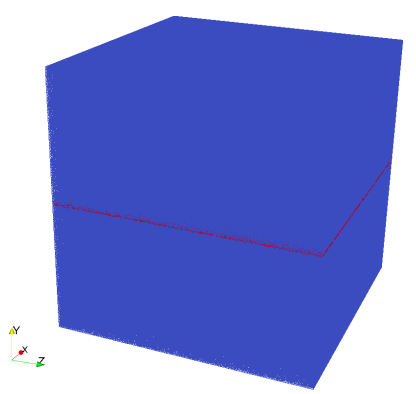

Figure 3: $100 \times 100 \times 100 \mathrm{~mm}$ cubic domain with $\kappa$ equals to 0 : the crack (colored in red) is in the plane $\mathrm{Y}=$ $50 \mathrm{~mm}$

The numerical results are illustrated in Figures 4 and 5. They show the components of the mean diffusivity tensor $\underline{\mathrm{D}}$ as a function of the crack width $[|u|]$ for the $\overline{\overline{10}} 0 \times 100 \times 100 \mathrm{~mm}$ cubic domain.

The anisotropy of the mean diffusivity tensor can be observed in Figure 4 since the diagonal components of $\underline{\underline{D}}$ do not increase in value with the same magnitude. $D_{x x}$ and $D_{z z}$ have the same value and increasing because the macroscopic crack is perpendicular to the $Y$-direction. In contrast, $D_{y y}$ remains invariant to the crack width. The value of this component is equal to the one for an homogeneous domain, namely $1.8 \times 10^{-12} \mathrm{~m}^{2} / \mathrm{s}$. These results are consistent with the work of ([17]).

Figure 5 plots the off-diagonal components of $\underline{\underline{\mathrm{D}}}$. Physically speaking, these terms correspond to crossed-interactions. For instance, $D_{x y}$ is the value of $\underline{\underline{\mathrm{D}}}$ in the $X$-direction when a gradient of concentration is applied in the $Y$ direction. $D_{x y}$ and $D_{z y}$ have the same level of magnitude in terms of value whereas $D_{x z}$ has higher value. This result is consistent regarding the location of the crack, namely in the $X-Z$ plane.

For sake of clarity, we present the form of $\underline{\underline{\mathrm{D}}}$ for different crack width $[|u|]$ corresponding to the three different ranges defined by equation (8) (we have omitted $\times 10^{-12} \mathrm{~m}^{2} / \mathrm{s}$ due to the lack of space in the column):

$$
\underline{\underline{\mathrm{D}}}^{0 \mu m}=\left(\begin{array}{ccc}
1.78 & 1.45 \times 10^{-5} & -4.51 \times 10^{-6} \\
1.45 \times 10^{-5} & 1.77 & 5.48 \times 10^{-6} \\
-4.51 \times 10^{-6} & 5.45 \times 10^{-6} & 1.77
\end{array}\right)
$$




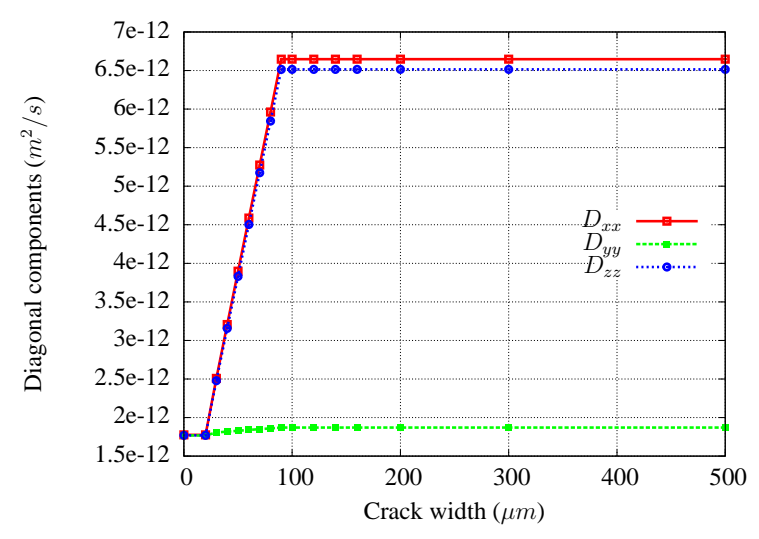

Figure 4: Diagonal components of $\underline{\underline{D}}$ in relation with the crack width

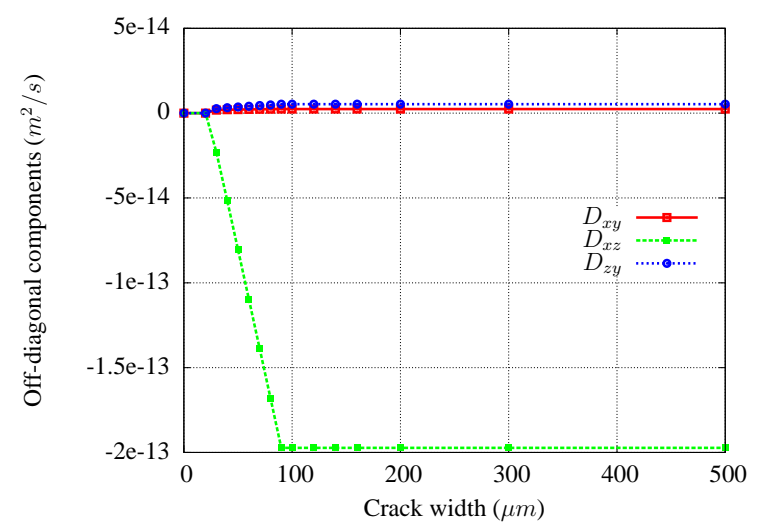

Figure 5: Off-diagonal components of $\underline{\underline{D}}$ in relation with the crack width

$\underline{\underline{\mathrm{D}}}^{30 \mu m}=\left(\begin{array}{ccc}2.51 & 1.79 \times 10^{-3} & -2.30 \times 10^{-2} \\ 1.79 \times 10^{-3} & 1.81 & 2.67 \times 10^{-3} \\ -2.30 \times 10^{-2} & 2.67 \times 10^{-3} & 2.48\end{array}\right)$

$\underline{\underline{\mathbf{D}}}^{90 \mu m}=\left(\begin{array}{ccc}6.65 & 2.41 \times 10^{-3} & -1.97 \times 10^{-1} \\ 2.41 \times 10^{-3} & 1.87 & 5.27 \times 10^{-3} \\ -1.97 \times 10^{-1} & 5.27 \times 10^{-3} & 6.51\end{array}\right)$

In respect of the form of $\underline{\underline{D}}$, we conclude that it is symmetric with real coefficients so it is diagonalisable. We find the sets of following eigenvalues $(1.78,1.77,1.77)^{0 \mu m},(2.52,1.81$, $2.47)^{30 \mu m}$ and $(6.79,1.87,6.37)^{90 \mu m}$ (note that we have omitted $\left.\times 10^{-12} \mathrm{~m}^{2} / \mathrm{s}\right)$. They are all positive so $\underline{\underline{D}}$ is positive-definite.

The eigenvalues of $\underline{\underline{\mathbf{D}}}^{0 \mu m}$ and $\underline{\underline{\mathbf{D}}}^{30 \mu m}$ are equal to the diagonal values of $\underline{\underline{D}}^{0 \mu m}$ and $\underline{\underline{D}}^{30 \mu m}$. Consequently, the off-diagonal terms of $\underline{\underline{\mathbf{D}}}^{0 \mu m}$ and $\underline{\underline{\mathbf{D}}}^{30 \mu m}$ are negligeable (terms in $10^{-14} \mathrm{~m}^{2} / \mathrm{s}$ and
$10^{-15} \mathrm{~m}^{2} / \mathrm{s}$ ). However for $\underline{\mathbf{D}}^{90 \mu m}$, this conclusion cannot hold. We conclude that when the crack width increases, the induced-anisotropy of $\underline{\underline{\mathrm{D}}}$ increases. This is accompanied with non negligeable off-diagonal terms.

Last but not least, the two important properties of $\underline{\underline{D}}$ aforementioned are checked: $\underline{\underline{D}}$ is symmetric and positive-definite (positive eigenvalues).

These two points are consistent with the theoretical results of ([20]). They assess the numerical accuracy of the upscaling method presented in Section 4 and its implementation in the context of the Finite Element Method.

The numerical examples presented above consider an artificial crack as heterogeneity. In this part, we propose a step beyond for the numerical simulations, where both mechanically-induced cracks and aggregates are considered. We first present the mechanical results and then we turn to the mean diffusivity tensor determination. Note that the mesoscale coupling is performed again by means of the experimental law of ([6]) presented in Section 3 and already used in the first simulation. As we will see hereafter this law is slightly modified to account for a two-phase material.

\subsection{Two-phase mechanically-induced cracked domain}

\subsubsection{Tensile test simulation:}

The $100 \times 100 \times 100 \mathrm{~mm}$ cubic domain is again regarded. It is composed this time of a mortar matrix with $35 \%$ of spherical aggregates. Two aggregate diameters $\Phi$ are regarded: 4 and 16 $\mathrm{mm}$. Cracking is mechanically-induced by a tensile test. Once again, the numerical model presented in Section 2 is used for the simulation of the mechanical problem.

Considering a two-phase material where stiff aggregates are embedded into a mortar matrix, we remind that three sets of elements are present in the simulation: those entirely lying inside the matrix or inside the aggregates (with the same elastic modulus and no strain discontinuity) and those that are split by a physical interface and 
whose strain discontinuity is activated.

Table 1 summarizes the mesoscale material properties pertaining to the mechanical model, for the different phases of the domain. Note that the aggregates are stiffer than the mortar matrix and remain in the elastic regime. The computation is made under displacement control according to the second spatial axis $Y$. We obtain a macroscale Young modulus and a macroscale limit stress equal to 29060 (28700) $\mathrm{MPa}$ and 2.05 (2.10) $\mathrm{MPa}$ for $\Phi$ equals to 4 (16) $\mathrm{mm}$, respectively.

\begin{tabular}{|l|l|l|l|}
\hline phase & $\begin{array}{l}E \\
(G P a)\end{array}$ & $\begin{array}{l}\sigma_{u} \\
(M P a)\end{array}$ & $\begin{array}{l}G_{f} \\
\left(\mathrm{~J} / \mathrm{m}^{2}\right)\end{array}$ \\
\hline $\begin{array}{l}\text { mortar } \\
\text { matrix }\end{array}$ & 18 & 3 & 5 \\
\hline aggregates & 127 & elastic & elastic \\
\hline interfaces & - & 2 & 5 \\
\hline
\end{tabular}

Table 1: Mesoscale material properties for the numerical simulations

The crack evolution for the cubic domain is shown in Figures 6 and 7 for $\Phi$ equals to 4 and $16 \mathrm{~mm}$, respectively. It corresponds to the microcracked bar elements for which the strong discontinuity has been activated. The crack is initiated around the aggregates in the interface elements corresponding to a weak zone (Figures 6(a) and 7(a)). As the applied macroscale strain increases, this crack is bridged over around the elastic aggregates (Figures 6(b) and 7(b)) leading to a kind of macro-crack in the direction roughly orthogonal to the imposed displacement and passing around the aggregates (Figures 6(c) and $7(\mathrm{c}))$.

Finally, we can conclude that (1) the maximum crack opening values are very close whatever the size of the aggregates is, (2) regarding Figures 6(c) and 7(c), the macro-crack pattern is more tortuous for an aggregate diameter equals to $16 \mathrm{~mm}$ and is rotated around the $X$ and $Z$ axis. Last but not least, one macro-crack is sufficient to drive the macroscale response into the softening regime.

Having in hands the results of the mechanical simulation, we now turn to the mean diffu-

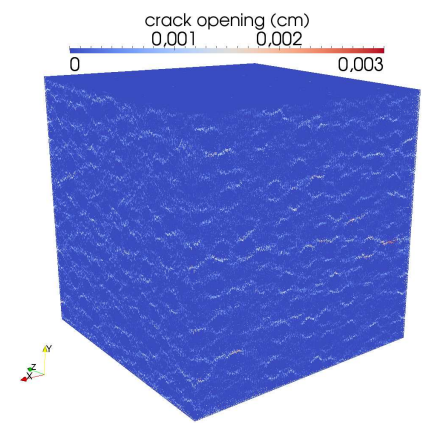

(a) Micro-crack initiation around the aggregates

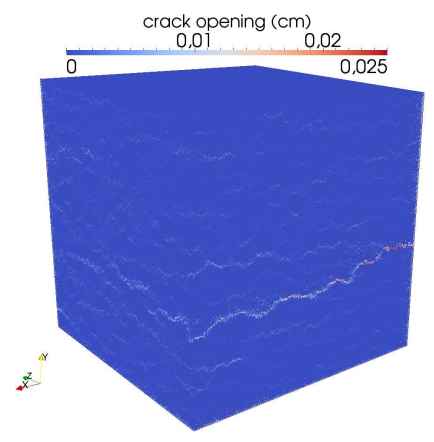

(b) Micro-crack coalescence process

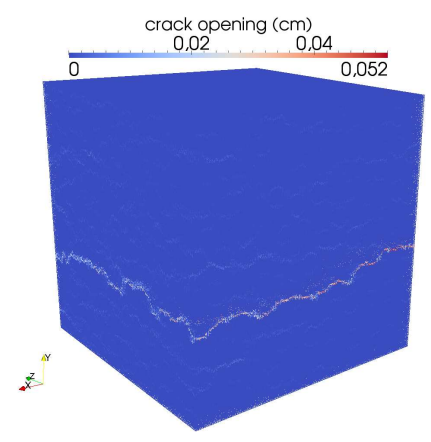

(c) Macro-crack formation

Figure 6: Crack pattern evolution and crack width values for the tensile test with $\Phi$ equals to $4 \mathrm{~mm}$ 


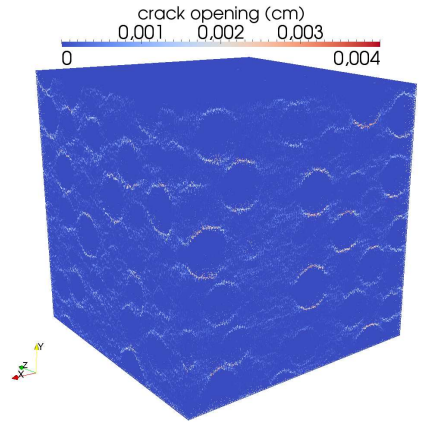

(a) Micro-crack initiation around the aggregates

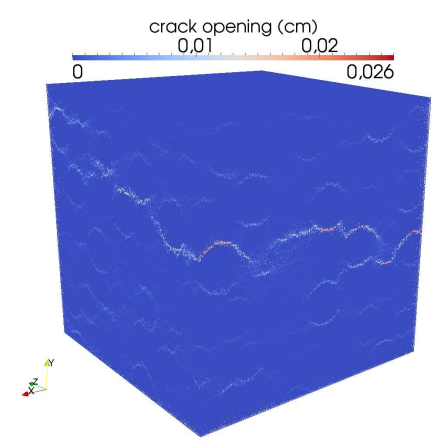

(b) Micro-crack coalescence process

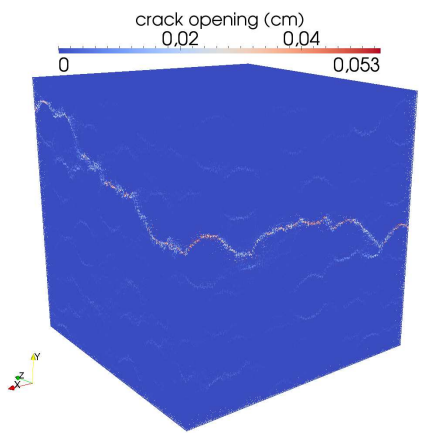

(c) Macro-crack formation

Figure 7: Crack pattern evolution and crack width values for the tensile test with $\Phi$ equals to $16 \mathrm{~mm}$ sivity tensor computation as presented in Section 4.

\subsubsection{Mean diffusivity tensor computation:}

First of all, the experimental law of ([6]) is slightly modified to account for a two-phase material such as:

$$
\left\{\begin{array}{rr}
D_{c r}\left(m^{2} / s\right)= & \theta D_{1}+(1-\theta) D_{2}, \\
\text { when }[|u|]<30 \mu m \\
D_{c r}\left(m^{2} / s\right)= \\
\\
D_{c r}\left(m^{2} / s\right)= \\
\text { when } 30 \mu m \leq 10^{-11}[|u|]-4 \times 10^{-10}, \\
14 \mid] \leq 80 \mu m \\
14 \times 10^{-10}, \\
\text { when }[|u|]>80 \mu m
\end{array}\right.
$$

where $D_{1}$ and $D_{2}$ are the diffusion coefficient in the mortar matrix and in the aggregates, respectively and $\theta$, the spatial position of the interface in an element computing in the meshing process (see ([1]) for details). Note that when an element is in the mortar matrix, $D_{1}$ is equal to $D_{2}$ and the original expression of ([6]) is found again.

Models parameters pertaining to the diffusion problem are given in Table 2. The aggregates are chosen impermeable such as their diffusion coefficient is $D_{2}=D_{1} / 100$. Note that $D_{1}$ has the value of $1.8 \times 10^{-12} \mathrm{~m}^{2} / \mathrm{s}$ and corresponds to the mortar matrix diffusion coeffficient.

\begin{tabular}{|l|l|}
\hline phase & $D_{m}\left(\mathrm{~m}^{2} / \mathrm{s}\right)$ \\
\hline $\begin{array}{l}\text { mortar ma- } \\
\text { trix }\end{array}$ & $\begin{array}{l}\text { equ. }(17): D_{1}=D_{2}=1.8 \times \\
10^{-12}\end{array}$ \\
\hline aggregates & $D_{2}=1.8 \times 10^{-14}$ \\
\hline interfaces & $\begin{array}{l}\text { equ. }(17): D_{1}=1.8 \times 10^{-12} \\
\text { and } D_{2}=1.8 \times 10^{-14}\end{array}$ \\
\hline
\end{tabular}

Table 2: Mesoscale material properties for the numerical simulations

Figure 8 shows the diagonal components of the mean diffusivity tensor as a function of the maximum crack width and the aggregates size.

Firstly, we can note a threshold value in terms of the maximum crack width around $0.25 \mathrm{~mm}$ corresponding to the micro-crack coalescence 
process leading to the macro-crack (see Figures 6(b) and 7(b) ). Around this threshold, the localized macro-crack takes over the advantage from the diffuse cracks in the bulk, in terms of path flow. This macro-crack gives an increase in the values of the diagonal components of the mean diffusivity tensor as observed in Figure 8.

Secondly, we note an increase of $8 \%, 16.4$ $\%$ and $4.7 \%$ between $D_{x x, 4 m m}$ and $D_{x x, 16 m m}$, $D_{y y, 4 m m}$ and $D_{y y, 16 m m}, D_{z z, 4 m m}$ and $D_{z z, 16 m m}$, respectively. Note that these values are only given for the last time step corresponding to a maximum crack opening equals to $0.52 \mathrm{~mm}$ for $\Phi$ equals to $4 \mathrm{~mm}$ and $0.53 \mathrm{~mm}$ for $\Phi$ equals to $16 \mathrm{~mm}$. Indeed this comparison holds only if the crack opening are very close. This increase in the diagonal components for $\Phi$ equals to 16 $m m$ could be explained by the rotation of the macro-crack around the $X$ and $Z$ axis with a rotation more important around the $X$ axis. Because the quantification of this rotation is not easy, the proposed explanation has to be seen as more qualitative than quantitative. Nevertheless the authors have proved the capability of the proposed method, even in the context of mechanically-induced cracks and aggregates, to determine macroscopic diffusivity tensors.

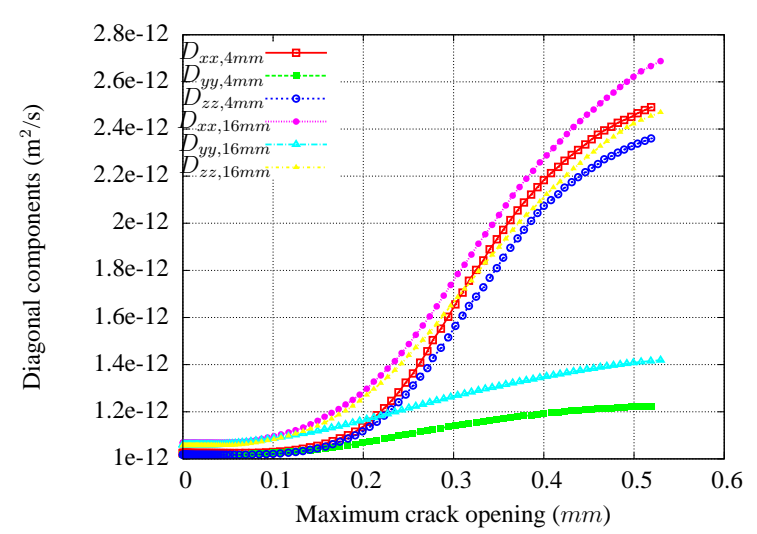

Figure 8: Diagonal components in relation with the maximum crack width and the aggreagtes size

\section{CONCLUSIONS}

We have presented in this paper a meso-macro numerical approach accounting for crack-induced diffusivity in heterogeneous materials. This nu- merical approach relies on (1) a mesoscale model where the diffusion coefficient is linked to the crack opening and (2) a macroscale model where a macroscopic concentration gradient is linked to a macroscopic flux, in the spirit of the porous media flow theory. The information from the mesoscale to the macroscale are upscaled by means of an homogeneisation method based on the work of ([20]). This presented framework leads to the computation of a macroscopic diffusivity tensor accounting for the evolution from diffuse cracks in the bulk to localized macro-crack(s), the tortuosity of the crack, and the induced-anisotropy.

The Strong Discontinuity Approach is the key point of this work in regards with the mesoscale coupling. This approach is an elegant method to model cracks within a medium. In addition, it gives the crack opening values for each Finite Element, at each time step. At the mesoscale, each crack is a path for an oriented flow in which the coefficient of diffusion follows the experimental law of ([6]). We have presented several numerical examples showing the ability of the numerical upscaling process to model anisotropic macroscopic diffusivity tensor. These examples confirm the intuitive idea that the whole set of fine cracks cannot lead to an isotropic diffusivity tensor.

\section{REFERENCES}

[1] N. Benkemoun, M. Hautefeuille, J.-B. Colliat, and A. Ibrahimbegovic. Modeling heterogeneous materials failure: 3D meso-scale models with embedded discontinuities. International Journal of Numerical Methods in Engineering, 82:1671-1688, 2010.

[2] R. I. Borja and J. E. Andrade. Critical state plasticity, part VI: Meso-scale finite element simulation of strain localization in discrete granular materials. Computer Methods in Applied Mechanics and Engineering, 195:5115-5140, 2006.

[3] B. S̆avija, M. Luković, and E. Schlangen. Lattice modeling of rapid chloride migra- 
tion in concrete. Cement and Concrete Research, 61-62(0):49-63, 2014.

[4] B. S̆avija, J. Pacheco, and E. Schlangen. Lattice modeling of chloride diffusion in sound and cracked concrete. Cement and Concrete Composites, 42(0):30 - 40, 2013.

[5] M. Şahmaran. Effect of flexure induced transverse crack and self-healing on chloride diffusivity of reinforced mortar. Journal of Materials Science, 42(22):91319136, 2007.

[6] A. Djerbi, S. Bonnet, and A. Khelidj. Influence of traversing crack on chloride diffusion into concrete. Cement and Concrete Research, 38 (6):877-883, 2008.

[7] A. Djerbi-Tegguer, S. Bonnet, A. Khelidj, and V. Baroghel-Bouny. Effect of uniaxial compressive loading on gas permeability and chloride diffusion coefficient of concrete and their relationship. Cement and Concrete Research, 52:131-139, 2013.

[8] B. Gérard and J. Marchand. Influence of cracking on the diffusion properties of cement-based materials: Part i: Influence of continuous cracks on the steady-state regime. Cement and Concrete Research, 30(1):37-43, 2000.

[9] P. Grassl. A lattice approach to model flow in cracked concrete. Cement and Concrete Composites, 31(7):454-460, 2009.

[10] M. Ismail, A. Toumi, R. François, and R. Gagné. Effect of crack opening on the local diffusion of chloride in cracked mortar samples. Cement and Concrete Research, 38(8 - 9):1106 - 1111, 2008.

[11] S.Y. Jang, B.S. Kim, and B.H. Oh. Effect of crack width on chloride diffusion coefficients of concrete by steady-state migration tests. Cement and Concrete Research, 41(1):9-19, 2011.
[12] W. L. Jin, Y. D. Yan, and H. L. Wang. Chloride diffusion in the cracked concrete. In : Oh, B.H., et al. (Eds), Fracture Mechanics of Concrete and Concrete Structures-Assessment, Durability, Monitoring and Retrofiting of Concrete Structures. Korea Concrete Institute, Seoul, p.880-886.

[13] X. Jourdain, J.-B. Colliat, C. DeSa, F. Benboudjema, and F. Gatuingt. Upscaling permeability for fractured concrete: mesomacro numerical approach coupled to strong discontinuities. International Journal for Numerical and Analytical Methods in Geomechanics, 38(5):536550, 2014.

[14] A. Lachihab and K. Sab. Aggregate composites: a contact based modeling. Computational Material Science, 33:467-490, 2005.

[15] R.W. Lewis, P. Nithiarasu, and Seetharamu K.N. Fundamentals of the finite element method for heat ans fluid flow. Taylor and Francis, New York, 2004.

[16] N. Moës, M. Cloirec, P. Cartraud, and J.F. Remacle. A computational approach to handle complex microstructure geometries. Computer Methods in Applied Mechanics and Engineering, 192:31633177, 2003.

[17] F. Nilenius, F. Larsson, K. Lundgren, and K. Runesson. Mesoscale modelling of crack-induced diffusivity in concrete. Computational Mechanics, 55(2):359370, 2015.

[18] M. Ortiz, Y. Leroy, and A. Needleman. A finite element method for localized failure analysis. Computer Methods in Applied Mechanics and Enginnering, 61:189-214, 1987. 
[19] E. Poulsen and L. Mejibro. Diffusion of chloride in concrete: theory ans application. Taylor and Francis, New York (USA), 2006.

[20] A. Pouya and A. Courtois. Définition de la perméabilité équivalente des massifs fracturés par des méthodes d'homogénéisation. Comptes Rendus Geoscience, 334(13):975 - 979, 2002.

[21] A. Pouya and O. Fouché. Permeability of 3D discontinuity networks: New tensors from boundary-conditioned homogenisation. Advances in Water Resources, 32(3):303 - 314, 2009.

[22] Jean-Marie Reynouard, Gilles PijaudierCabot, and Jean-Michel Torrenti. Mechanical behavior of concrete. Wiley, New-York, 2009.

[23] E. Schlangen. Experimental and numerical analysis of fracture processes in concrete. TU Delft, Delft University of Technology, 1993.

[24] E. Schlangen and E. J. Garboczi. Fracture simulations of concrete using lattice models: computational aspects. Engineering Fracture Mechanics, 57:319-332, 1997.

[25] E. Schlangen and J. G. M. van Mier. Simple lattice model for numerical simulation of fracture of concrete materials and structures. Materials and Structures, 25:534542, 1992.

[26] J.C. Simo and T.J.R. Hughes. Computational Inelasticity. Interdisciplinary Applied Mathematics. Springer Verlag, New York, Berlin, Heidelberg, 1997.

[27] J.C. Simo, J. Oliver, and F. Armero. An analysis of strong discontinuities induced by strain-softening in rate independent inelastic solids. Computational Mechanics, 12:277-296, 1993.
[28] J.C. Simo and M. Rifai. A class of mixed assumed strain methods and the method of incompatible modes. International Journal of Numerical Methods in Engineering, 29:1595-1638, 1990.

[29] L. Wang and T. Ueda. Mesoscale modelling of the chloride diffusion in cracks and cracked concrete. Journal of $A d$ vanced Concrete Technology, 9(3):241249, 2011.

[30] K. Washizu. Variational methods in elasticity and plasticity. Pergamon Press, New York, 3 edition, 1982.

[31] E.L. Wilson. The static condensation algorithm. International Journal for Numerical Methods in Engineering, 8:198-203, 1974.

[32] P. Wriggers and S. O. Moftah. Mesoscale models for concrete: Homogenisation and damage behaviour. Finite Elements in Analysis and Design, 42:623-636, 2006.

[33] M. Yip, J. Mohle, and J. E. Bolander. Automated modeling of three-dimensional structural components using irregular lattices. Computer-Aided Civil anf Infrastructure Engineering, 120:393-407, 2005.

[34] I.S. Yoon, E. Schlangen, M.R. de Rooij, and K. van Breugel. The effect of cracks on chloride penetration into concrete. Key Engineering Materials, 348349:769-772, 2007. 